\title{
Finite Dimensional Representations of the Quantum Lorentz Group
}

\author{
Mitsuhiro Takeuchi \\ Institute of Mathematics, University of Tsukuba, Tsukuba, Ibaraki 305, Japan
}

Received March 28, 1991

\begin{abstract}
All finite dimensional irreducible representations of the quantum Lorentz group $\mathrm{SL}_{q}(2, \mathbb{C})$ are described explicitly and it is proved all finite dimensional representations of $\mathrm{SL}_{q}(2, \mathbb{C})$ are completely reducible. The conjecture of Podles and Woronowicz will be answered affirmatively.
\end{abstract}

\section{Introduction}

The quantum Lorentz group $\mathrm{SL}_{q}(2, \mathbb{C})$, where $q$ is a real parameter $\neq 0, \pm 1$, was introduced by Podleś and Woronowicz [PW], and the Iwasawa decomposition and representation theory were studied. This quantum group is combined with the double group of $\mathrm{SU}_{q}(2)$, a $q$-analogue of the compact group $\mathrm{SU}(2)$ [W, MMNNU], through the Iwasawa decomposition. Let $A_{q}$ (respectively $B_{q}$ ) be the *-Hopf algebra corresponding to the quantum group $\mathrm{SL}_{q}(2, \mathbb{C})$ (respectively $\mathrm{SU}_{q}(2)$ ). (A *-Hopf algebra means a Hopf algebra over $\mathbb{C}$ with $\mathrm{a}^{*}$-operation satisfying some properties. See Sect. 4.) The dual vector space $B_{q}^{\prime}=\operatorname{Hom}_{\mathbb{C}}\left(B_{q}, \mathbb{C}\right)$ has a topological Hopf algebra structure. By a topological Hopf algebra, we mean a topological analogue of the usual Hopf algebra, in which the underlying vector space is assumed to have a linear topology and the complete tensor product $\hat{\otimes}$ plays the role of the usual tensor product. (See Sect. 1.)

Podleś and Woronowicz have introduced some topological Hopf algebra structure as well as some *-operation on $B_{q} \hat{\otimes} B_{q}^{\prime}$ and have proved there is an injective *-Hopf algebra map of $A_{q}$ into $B_{q} \widehat{\otimes} B_{q}^{\prime}$. We call

$$
\mathscr{E}_{q}=B_{q} \hat{\otimes} B_{q}^{\prime}
$$

the quantum double of $B_{q}$. This is the dual version of Drinfeld's quantum double [D], and corresponds to the double group of $\mathrm{SU}_{q}(2)$.

The topological Hopf algebra $\mathscr{E}_{q}$ has the largest (non-topological) Hopf subalgebra $E_{q}$, and what they have done is the construction of an injective *-Hopf algebra map of $A_{q}$ into $E_{q}$. This is not surjective. There is a central group-like 
element $\tilde{\tau}$ of order 2 in $E_{q}$ outside $A_{q}$. The conjecture $6.4[\mathrm{PW}$ ] tells that we have

$$
E_{q}=A_{q} \otimes A_{q} \tilde{\tau} .
$$

The purpose of this paper is to prove this conjecture is true.

This conjecture has a close relation with representation theory. By a representation of the quantum Lorentz group $\mathrm{SL}_{q}(2, \mathbb{C})$ we mean a (right) comodule for $\mathscr{E}_{q}$. This concept involves a linear topology, since $\mathscr{E}_{q}$ is a topological Hopf algebra, but we consider only discrete comodules. Thus the structure map of a comodule $V$ is a linear map $\rho: V \rightarrow V \hat{\otimes} \mathscr{E}_{q}$ with discrete topology on $V$. We see $\rho(V)$ is contained in $V \otimes E_{q}$ if and only if $V$ is locally finite, i.e., it is the sum of finite dimensional subcomodules. In other words, locally finite representations of $\mathrm{SL}_{q}(2, \mathbb{C})$ are the same thing as comodules for $E_{q}$.

In the previous paper [T2], we showed how the finite dimensional representation theory of $U_{q}=\mathrm{U}_{q}(\mathfrak{s l}(2))$ leads to an explicit description of the dual Hopf algebra $U_{q}^{\circ}$ which is the largest (non-topological) Hopf subalgebra of the topological dual $U_{q}^{\prime}$. One sees we are precisely in the same situation.

We will think $A_{q}$ is a Hopf subalgebra of $E_{q}$. Comodules for $A_{q}$ are called smooth representations of $\mathrm{SL}_{q}(2, \mathbb{C})$. The above conjecture literally tells that if $V$ is a comodule for $E_{q}$, there are smooth representations $V_{1}$ and $V_{2}$ (uniquely determined up to isomorphisms) such that

$$
V \cong V_{1} \oplus\left(V_{2} \otimes \mathbb{C} \tilde{\tau}\right) .
$$

One sees this is equivalent to the previous statement.

To prove the conjecture, first we observe that comodules for $\mathscr{E}_{q}$ are the same thing as crossed bimodules for $B_{q}$ in the sense of Yetter [Y]. Next, we note that there is an isomorphism of coalgebras (but not Hopf algebras)

$$
B_{q} \otimes B_{q^{-1}} \cong A_{q} .
$$

This means all comodules for $A_{q}$, i.e., all smooth representations, are completely reducible, and if $V_{k}$ (respectively $V_{k}^{*}$ ) denotes the $k+1$-dimensional simple comodule for $B_{q}$ (respectively $B_{q^{-1}}$ ), then $V_{k} \otimes V_{l}^{*}, k, l \in \mathbb{N}$, give all mutually non-isomorphic simple comodules for $A_{q}$. (See [PW, Theorem 6.3].)

Therefore, the conjecture reduces to the following theorems.

Theorem A. $V_{k} \otimes V_{l}^{*}$ and $V_{k} \otimes V_{l}^{*} \otimes \mathbb{C} \tilde{\tau}, \quad k, l \in \mathbb{N}$, give a complete set of representatives for the isomorphism classes of all finite dimensional simple crossed bimodules for $B_{q}$.

Theorem B. All finite dimensional crossed bimodules for $B_{q}$ are completely reducible.

The paper is outlined as follows. In Sect. 1, we state basic facts on topological Hopf algebras. In Sect. 2, we review the construction of the quantum double. In Sect. 3, we establish the correspondence between comodules for the quantum double and crossed bimodules. In Sect. 4, we review the construction of the quantum Lorentz group $\mathrm{SL}_{q}(2, \mathbb{C})$ as well as the description of *-Hopf algebras $A_{q}$ and $B_{q}$ and the embedding of $A_{q}$ into the quantum double of $B_{q}$. In Sect. 5, we express the crossed axiom for $B_{q}$ in terms of $\left(U_{q}, B_{q}\right)$-bimodules. Using this expression, we prove Theorem A in Sect. 6 and Theorem B in Sect. 7. The final section, Sect. 8, which is an appendix, describes the braid structure on $A_{q}$ arising from the braid structure of the category of crossed bimodules. 
The first three sections deal with generalities, and we work over a general field $k$. In the rest of the sections we let $k=\mathbb{C}$ the complex numbers.

The symbol * is limited to mean the *-operation. Hence we denote by $V^{\prime}$ the dual vector space $\operatorname{Hom}_{k}(V, k)$ (not by $\left.V^{*}\right)$.

\section{Topological Hopf Algebras}

We state basic facts on topological Hopf algebras. Generalities on topological coalgebras are developed in [T1].

We work over a field $k$. A topology on a vector space $V$ is linear if all translation $v+(v \in V)$ is continuous and if there is a fundamental system of neighborhoods of $0\left\{V_{\alpha}\right\}$ consisting of vector subspaces. In this paper, we consider only Hausdorff topological vector spaces. This is equivalent to saying $\bigcap_{\alpha} V_{\alpha}=0$. The completion of $V$ is defined to be

$$
\hat{V}=\underset{\alpha}{\lim } V / V_{\alpha}
$$

which has the prodiscrete topology.

If $V$ and $W$ are topological vector spaces with fundamental systems of neighborhoods of $0\left\{V_{\alpha}\right\}$ and $\left\{W_{\beta}\right\}$ we give the linear topology on $V \otimes W$ such that $\left\{V \otimes W_{\beta}+V_{\alpha} \otimes W\right\}$ is a fundamental system of neighborhoods of $0 . V \otimes W$ is Hausdorff if $V$ and $W$ are Hausdorff. The completion of $V \otimes W$ will be denoted $V \hat{\otimes} W$, and this plays a basic role in the paper. Note that we have

$$
V \hat{\otimes} W=\lim _{\alpha, \beta} V / V_{\alpha} \otimes W / W_{\beta}
$$

We always give the discrete topology on $k$.

If $M$ is a vector space, we give the linear topology on the dual space $M^{\prime}$ such that $L^{\perp}$ for all finite dimensional subspaces $L$ of $M$ form a fundamental system of neighborhoods of 0 . More generally, if $M$ and $N$ are vector spaces, $\operatorname{Hom}_{k}(M, N)$ will be given the linear topology such that $\operatorname{Hom}_{k}(M / L, N)$ for all finite dimensional $L$ form a fundamental system of neighborhoods of 0 . There is a natural isomorphism of topological vector spaces [T1, p. 513],

$$
N \hat{\otimes} M^{\prime} \cong \operatorname{Hom}_{k}(M, N) \text {, }
$$

where $N$ is given the discrete topology.

By a topological coalgebra we mean a topological vector space $\mathscr{C}$ with linear continuous maps

$$
\Delta: \mathscr{C} \rightarrow \mathscr{C} \hat{\otimes} \mathscr{C}, \quad \varepsilon: \mathscr{C} \rightarrow k
$$

satisfying the coassociativity and the counit condition [T1, p. 510].

A topological algebra, its dual concept, consists of a topological vector space $\mathscr{A}$ and linear continuous maps

$$
m: \mathscr{A} \hat{\otimes} \mathscr{A} \rightarrow \mathscr{A}, \quad u: k \rightarrow \mathscr{A} .
$$

If $A$ is an algebra and $C$ is a coalgebra, then the dual topological vector spaces $A^{\prime}$ and $C^{\prime}$ have natural structures of a topological coalgebra and a topological 
algebra respectively. More generally, the topological vector space $\mathscr{A}=\operatorname{Hom}_{k}(C, A)$ has the following topological algebra structure:

$$
\begin{aligned}
\tilde{m} & =\operatorname{Hom}_{k}(\Delta, m): \mathscr{A} \hat{\otimes} \mathscr{A} \rightarrow \mathscr{A}, \\
\tilde{u} & =\operatorname{Hom}_{k}(\varepsilon, u): k \rightarrow \mathscr{A},
\end{aligned}
$$

where we identify $\mathscr{A} \widehat{\otimes} \mathscr{A}=\operatorname{Hom}_{k}(C \otimes C, A \otimes A)[\mathrm{T} 1,1.14]$. The multiplication $\tilde{m}$ is called the convolution product [S, pp. 69-72]. If we consider $A$ is a discrete topological algebra, we have an isomorphism of topological algebras

$$
\mathscr{A} \cong A \hat{\otimes} C^{\prime} \text {. }
$$

If $H$ is a bialgebra, $\operatorname{End}_{k}(H)$ has two algebra structures. The convolution product will be written as $f \cdot g$, while the composition will be written as $f \circ g$ or $f g$. Note that we have an isomorphism of topological algebras (with convolution products)

$$
\operatorname{End}_{k}(H \otimes H) \cong \operatorname{End}_{k}(H) \hat{\otimes} \operatorname{End}_{k}(H) .
$$

Let $\mathscr{C}$ be a topological coalgebra. Since we assume it is Hausdroff, $\mathscr{C} \otimes \mathscr{C}$ is a subspace of $\mathscr{C} \hat{\otimes} \mathscr{C}$. A subspace $C$ of $\mathscr{C}$ is called a subcoalgebra if we have $\Delta(C) \subset C \otimes C$. (We consider no topology on $C$.) Obviously the sum of all subcoalgebras is the largest subcoalgebra of $\mathscr{C}$. For example, if $A$ is an algebra, the largest subcoalgebra of the topological coalgebra $A^{\prime}$ is denoted by $A^{\circ}$ [S, Chap. VI].

By a comodule for $\mathscr{C}$, we mean a discrete vector space $V$ with a linear map

$$
\rho: V \rightarrow V \hat{\otimes} \mathscr{C}
$$

satisfying the usual axiom:

$$
\begin{aligned}
& (I \hat{\otimes} \Delta) \circ \rho=(\rho \hat{\otimes} I) \circ \rho \quad \text { as maps } \quad V \rightarrow V \hat{\otimes} \mathscr{C} \hat{\otimes} \mathscr{C} \\
& \text { and }(I \hat{\otimes} \varepsilon) \circ \rho \quad \text { is the canonical map } V \rightarrow V \hat{\otimes} k .
\end{aligned}
$$

All commodules are right in this paper. If $\mathscr{C}=A^{\prime}$ the dual topological coalgebra of an algebra $A$, then the comodules for $\mathscr{C}$ are naturally identified with the left $A$-modules [T1, Theorem 1.19].

1.1 Proposition. Let $\mathscr{C}$ be a complete topological coalgebra with the largest subcoalgebra $C$. Let $(V, \rho)$ be a comodule for $\mathscr{C}$. We have $\rho(V) \subset V \otimes C$ if and only if $V$ is locally finite, i.e., it is the sum of finite dimensional subcomodules.

Proof. The "only if" part follows from [S, Theorem 2.1.3]. To prove the "if" part, we may assume $V$ is finite dimensional. Note that $V \otimes \mathscr{C}=V \hat{\otimes} \mathscr{C}$, since $\mathscr{C}$ is complete. Take a base $v_{1}, \ldots, v_{n}$ for $V$ and write

$$
\rho\left(v_{j}\right)=\sum_{i} v_{i} \otimes x_{i j}
$$

It is easy to see

$$
\Delta\left(x_{i j}\right)=\sum_{s} x_{i s} \otimes x_{s j}
$$

hence $x_{i j}$ span a subcoalgebra and we have $\rho(V) \subset V \otimes C$. 
A topological bialgebra is the topological version of the concept of a bialgebra. The underlying vector space $\mathscr{H}$ is assumed to have a linear topology and the structure maps which are linear continuous

$$
\begin{array}{ll}
m: \mathscr{H} \hat{\otimes} \mathscr{H} \rightarrow \mathscr{H}, \quad u: k \rightarrow \mathscr{H}, \\
\Delta: \mathscr{H} \rightarrow \mathscr{H} \hat{\otimes} \mathscr{H}, \quad \varepsilon: \mathscr{H} \rightarrow k
\end{array}
$$

are assumed to satisfy the same axioms as a usual bialgebra. (Read $\hat{\otimes}$ for $\otimes$ in the diagrams of [S, p. 52].)

If $H$ is a bialgebra, then the topological dual vector space $H^{\prime}$ has a natural structure of a topological bialgebra.

For a topological bialgebra $\mathscr{H}$, let $E=\operatorname{End}_{\text {cont }}(\mathscr{H})$ be the vector space of all linear continuous endomorphisms. This space is closed relative to the convolution product

$$
f \cdot g=m \circ(f \hat{\otimes} g) \circ \Delta .
$$

$E$ is an algebra with this product and unit $u \varepsilon$. If the identity $I$ has an inverse $S$ in the algebra $E$, we say $\mathscr{H}$ is a topological Hopf algebra and $S$ the antipode. Just as the non-topological Hopf algebras [S, Proposition 4.0.1], one sees the antipode is an anti-endomorphism of the topological bialgebra.

1.2 Proposition. The largest subcoalgebra $H$ of a topological Hopf algebra $\mathscr{H}$ is a Hopf algebra.

This follows easily, since $k, H H$, and $S(H)$ are subcoalgebras, hence they are contained in $H$.

\section{Quantum Double}

Podleś and Woronowicz [PW, Sect. 4] introduced the dual concept of Drinfeld's quantum double [D] under the name "double group." The construction is reviewed in the context of topological Hopf algebras. We include all proofs for self-containedness.

Let $H$ be a Hopf algebra with structure maps $m, u, \Delta, \varepsilon$. Assume the antipode $S$ of $H$ is bijective. We will make the topological algebra $\mathscr{E}=\operatorname{End}_{k}(H)$ into a topological Hopf algebra. Let $\tilde{m}=\operatorname{Hom}_{k}(\Delta, m)$ and $\tilde{u}=\operatorname{Hom}_{k}(\varepsilon, u)$ be the structure maps of $\mathscr{E}$, and recall we have

$$
\mathscr{E} \otimes \mathscr{E}=\operatorname{End}_{k}(H \otimes H)
$$

as topological algebras (Sect. 1).

The comultiplication $\widetilde{\Delta}: \mathscr{E} \rightarrow \mathscr{E} \hat{\otimes} \mathscr{E}$ is defined to be the following continuous homomorphism of topological algebras:

$$
\begin{aligned}
& \tilde{\Delta}: \operatorname{End}_{k}(H) \rightarrow \operatorname{End}_{k}(H \otimes H) \\
& \tilde{\Delta}(f)=\sigma \cdot\left(\Delta \circ f \circ m^{o p}\right) \cdot \sigma^{-1},
\end{aligned}
$$

where $\sigma=(u \otimes I) \circ(I \otimes \varepsilon)$, hence $\sigma^{-1}=(u \otimes I) \circ S \circ(I \otimes \varepsilon)$. We have

$$
\tilde{\Delta}(f)(x \otimes y)=\sum\left(1 \otimes x_{(1)}\right) \Delta\left(f\left(y x_{(2)}\right)\right)\left(1 \otimes S\left(x_{(3)}\right)\right), \quad x, y \in H,
$$

where and in the following we use the sigma notation [S, p. 10]. 
2.1 Lemma. $\tilde{\Delta}$ is coassociative.

Proof. If $F \in \operatorname{End}_{k}(H \otimes H)$, then $x \otimes y \otimes z$ in $H \otimes H \otimes H$ is mapped by $(I \hat{\otimes} \tilde{\Delta})(F)$ to $\sum\left(1 \otimes 1 \otimes y_{(1)}\right)(I \otimes \Delta)\left(F\left(x \otimes z y_{(2)}\right)\right)\left(1 \otimes 1 \otimes S\left(y_{(3)}\right)\right)$,

and by $(\tilde{\Delta} \hat{\otimes} I)(F)$ to

$$
\sum\left(1 \otimes x_{(1)} \otimes 1\right)(\Delta \otimes I)\left(F\left(y x_{(2)} \otimes z\right)\right)\left(1 \otimes S\left(x_{(3)}\right) \otimes 1\right) .
$$

If $F=\tilde{\Delta}(f)$, both are equal to

$$
\sum\left(1 \otimes x_{(1)} \otimes y_{(1)} x_{(2)}\right) \Delta_{2}\left(f\left(z y_{(2)} x_{(3)}\right)\right)\left(1 \otimes S\left(x_{(5)}\right) \otimes S\left(y_{(3)} x_{(4)}\right)\right),
$$

where $\Delta_{2}: H \rightarrow H \otimes H \otimes H$ the iterated comultiplication.

Define the counit $\tilde{\varepsilon}: \mathscr{E}=\operatorname{End}_{k}(H) \rightarrow k$,

$$
\tilde{\varepsilon}(f)=\varepsilon(f(1)) .
$$

2.2 Theorem. $\mathscr{E}$ is a topological Hopf algebra.

Proof. The counit condition is easy to check. Define a continuous linear map $\widetilde{S}: \mathscr{E} \rightarrow \mathscr{E}$,

$$
\tilde{S}(f)=S \cdot\left(S \circ f \circ S^{-1}\right) \cdot I
$$

which maps $x$ in $H$ to $\sum S\left(x_{(1)}\right) S f S^{-1}\left(x_{(2)}\right) x_{(3)}$. We show $\tilde{S}$ is an antipode of $\mathscr{E}$. If $F \in \operatorname{End}_{k}(H \otimes H)$, then $(I \hat{\otimes} \widetilde{S})(F)$ maps $x \otimes y$ in $H \otimes H$ to

$$
\sum\left(1 \otimes S\left(y_{(1)}\right)\right)(I \otimes S) F\left(x \otimes S^{-1}\left(y_{(2)}\right)\right)\left(1 \otimes y_{(3)}\right) .
$$

If $F=\tilde{\Delta}(f)$, this becomes

$$
\sum\left(1 \otimes S\left(y_{(1)}\right) S^{2}\left(x_{(3)}\right)\right)(I \otimes S) \Delta\left(f\left(S^{-1}\left(y_{(2)}\right) x_{(2)}\right)\right)\left(1 \otimes S\left(x_{(1)}\right) y_{(3)}\right) .
$$

Hence $\tilde{m}(I \hat{\otimes} \tilde{S}) \tilde{\Delta}(f)$ maps $x$ in $H$ to

$$
m\left(\sum\left(1 \otimes S\left(x_{(4)}\right) S^{2}\left(x_{(3)}\right)\right)(I \otimes S) \Delta\left(f\left(S^{-1}\left(x_{(5)}\right) x_{(2)}\right)\right)\left(1 \otimes S\left(x_{(1)}\right) x_{(6)}\right)\right)
$$

which reduces to $\varepsilon(f(1)) \varepsilon(x)$. This means we have

$$
\tilde{m}(I \hat{\otimes} \tilde{S}) \tilde{\Delta}=\tilde{u} \tilde{\varepsilon} .
$$

Similarly, $(\tilde{S} \hat{\otimes} I) \tilde{\Delta}(f)$ maps $x \otimes y$ to

$$
\sum\left(S\left(x_{(1)}\right) \otimes S^{-1}\left(x_{(4)}\right)\right)(S \otimes I) \Delta\left(f\left(y S^{-1}\left(x_{(3)}\right)\right)\right)\left(x_{(5)} \otimes x_{(2)}\right) .
$$

Hence $\tilde{m}(\tilde{S} \hat{\otimes} I) \tilde{\Delta}(f)$ maps $x$ to

$$
\sum S\left(x_{(1)}\right) S\left(f\left(x_{(6)} S^{-1}\left(x_{(3)}\right)\right)_{(1)}\right) x_{(5)} S^{-1}\left(x_{(4)}\right) f\left(x_{(6)} S^{-1}\left(x_{(3)}\right)\right)_{(2)} x_{(2)}
$$

which is equal to $\varepsilon(f(1)) \varepsilon(x)$. This means we have

$$
\tilde{m}(\tilde{S} \hat{\otimes} I) \tilde{\Delta}=\tilde{u} \tilde{\varepsilon} .
$$

Hence $\tilde{S}$ is the antipode of $\mathscr{E}$. 
2.3 Definition. The topological Hopf algebra $\mathscr{E}$ is called the quantum double of $H$.

If we identify $\mathscr{E}=H \hat{\otimes} H^{\prime}$ as a topological algebra, the structure maps $\tilde{\Delta}, \tilde{\varepsilon}$, and $\tilde{S}$ have the following expressions:

$$
\begin{aligned}
& \tilde{\Delta}: H \hat{\otimes} H^{\prime} \stackrel{\Delta \hat{\otimes} \Delta^{\mathrm{op}}}{\longrightarrow}(H \otimes H) \hat{\otimes}\left(H^{\prime} \hat{\otimes} H^{\prime}\right) \stackrel{I \hat{\otimes} \omega \hat{\otimes} I}{\longrightarrow} \\
& \quad(H \otimes H) \hat{\otimes}\left(H^{\prime} \hat{\otimes} H^{\prime}\right) \stackrel{I \hat{\otimes} t w \hat{\otimes} I}{\longrightarrow}\left(H \hat{\otimes} H^{\prime}\right) \hat{\otimes}\left(H \hat{\otimes} H^{\prime}\right), \\
& \tilde{\varepsilon}: H \hat{\otimes} H^{\prime} \stackrel{\varepsilon \hat{\otimes} \varepsilon}{\longrightarrow} k \hat{\otimes} k=k, \\
& \tilde{S}: H \hat{\otimes} H^{\prime} \stackrel{\omega}{\longrightarrow} H \hat{\otimes} H^{\prime} \stackrel{s \hat{\otimes} s^{-1}}{\longrightarrow} H \hat{\otimes} H^{\prime},
\end{aligned}
$$

where $\omega$ denotes the inner action $f \mapsto I \cdot f \cdot S$. (We think $I, S$ are elements in $H \hat{\otimes} H^{\prime}$.) One sees our construction of the quantum double coincides with the double group [PW, Sect. 4].

It follows we have the following homomorphisms of topological Hopf algebras:

$$
\begin{aligned}
& \pi_{1}=I \hat{\otimes} \varepsilon: \mathscr{E}=H \hat{\otimes} H^{\prime} \rightarrow H, \\
& \pi_{2}=\varepsilon \hat{\otimes} I: \mathscr{E}=H \hat{\otimes} H^{\prime} \rightarrow H^{\prime c o p},
\end{aligned}
$$

where $H$ is given the discrete topology and cop means the coalgebra opposite.

2.6 Proposition. The composite

$$
\mathscr{E} \stackrel{\tilde{\Delta}}{\longrightarrow} \mathscr{E} \hat{\otimes} \mathscr{E} \stackrel{\pi_{1} \hat{\otimes} \pi_{2}}{\longrightarrow} H \hat{\otimes} H^{\prime}
$$

is equal to the identity.

Proof. If we identify $\mathscr{E} \hat{\otimes} \mathscr{E}=\operatorname{End}_{k}(H \otimes H)$ and $H \hat{\otimes} H^{\prime}=\operatorname{End}_{k}(H)$, then $\pi_{1} \hat{\otimes} \pi_{2}$ maps $F$ in $\operatorname{End}_{k}(H \otimes H)$ to the composite $(I \otimes \varepsilon) \circ F \circ(u \otimes I)$. If $F=\tilde{\Delta}(f)$, this composite reduces to $f$.

\section{Crossed Bimodules}

This section gives a correspondence between comodules for the quantum double and crossed bimodules in the sense of Yetter [Y].

Let $H$ be a Hopf algebra and let $\mathscr{E}=\operatorname{End}_{k}(H)$ its quantum double.

By a right bimodule for $H$, we mean a right $H$-module and a right $H$-comodule $V$. The coaction will be denoted [S, p. 32]

$$
v \mapsto \sum v_{(0)} \otimes v_{(1)}, \quad v \in V .
$$

3.1 Crossed Axiom [Y, Def. 3.6].

$$
\sum v_{(0)} h_{(1)} \otimes v_{(1)} h_{(2)}=\sum\left(v h_{(2)}\right)_{(0)} \otimes h_{(1)}\left(v h_{(2)}\right)_{(1)}, \quad v \in V, \quad h \in H .
$$

A crossed bimodule for $H$ means a right bimodule for $H$ satisfying the crossed axiom.

Let $V$ be a comodule for the topological bialgebra $\mathscr{E}$ with structure

$$
\rho: V \rightarrow V \hat{\otimes} \mathscr{E} .
$$


By means of the topological bialgebra maps $\pi_{1}, \pi_{2}(2.5)$, we have the following comodule structures:

$$
\begin{aligned}
& \rho_{1}: V \stackrel{\rho}{\longrightarrow} V \hat{\otimes} \mathscr{E} \stackrel{I \hat{\otimes} \pi_{1}}{\longrightarrow} V \otimes H, \\
& \rho_{2}: V \stackrel{\rho}{\longrightarrow} V \hat{\otimes} \mathscr{E} \stackrel{I \hat{\otimes} \pi_{2}}{\longrightarrow} V \hat{\otimes} H^{\prime \text { cop }} .
\end{aligned}
$$

The second comodule structure $\rho_{2}$ can be thought of as a right $H$-module structure. It follows from 2.6 Proposition that we have

$$
\rho: V \stackrel{\rho_{2}}{\longrightarrow} V \hat{\otimes} H^{\prime \text { cop }} \stackrel{\rho_{1} \hat{\otimes} I}{\longrightarrow} V \hat{\otimes} H \hat{\otimes} H^{\prime \text { cop }}=V \hat{\otimes} \mathscr{E} .
$$

Conversely, when $V$ is a right $H$-bimodule, define $\rho$ as the above composite.

3.2 Proposition. $(V, \rho)$ is a right comodule for $\mathscr{E}$ if and only if the crossed axiom holds for the bimodule $V$.

Proof. The counit condition for $\rho$ is always true. Identify

$$
V \hat{\otimes} \mathscr{E}=V \hat{\otimes} \operatorname{End}_{k}(H)=\operatorname{Hom}_{k}(H, V \otimes H)
$$

as topological vector spaces. To say $(V, \rho)$ is a comodule means that we have the following commutative diagram:

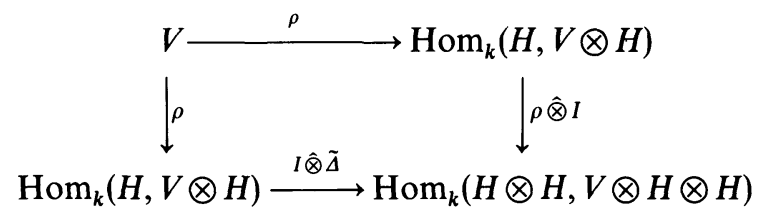

with comultiplication $\tilde{\Delta}$ of $\mathscr{E}$. Let $f=\rho(v)$ with $v \in V$. Then $f$ maps $h \in H$ to $\sum(v h)_{(0)} \otimes(v h)_{(1)}$. One sees by definition of $\tilde{\Delta}$

$$
\begin{aligned}
(\rho \hat{\otimes} I)(f)(x \otimes y) & =\sum \rho_{1}\left((v y)_{(0)} x\right) \otimes(v y)_{(1)}, \\
(I \hat{\otimes} \tilde{\Delta})(f)(x \otimes y) & =\sum\left(v y x_{(2)}\right)_{(0)} \otimes\left(1 \otimes x_{(1)}\right) \Delta\left(\left(v y x_{(2)}\right)_{(1)}\right)\left(1 \otimes S\left(x_{(3)}\right)\right)
\end{aligned}
$$

for $x, y \in H$. Note that both are functions of $v y$ and $x$. So, we may assume $y=1$, and the comodule condition tells that we have

$$
\sum\left(v_{(0)} x_{(1)}\right)_{(0)} \otimes\left(v_{(0)} x_{(1)}\right)_{(1)} \otimes v_{(1)} x_{(2)}=\sum\left(v x_{(2)}\right)_{(0)} \otimes\left(v x_{(2)}\right)_{(1)} \otimes x_{(1)}\left(v x_{(2)}\right)_{(2)}
$$

for all $v \in V, x \in H$. This follows from the crossed axiom by application of the map $\rho_{1} \otimes I$, and conversely, the crossed axiom follows from this by application of the map $I \otimes \varepsilon \otimes I$. Hence the comodule condition is equivalent to the crossed axiom.

It follows that the crossed bimodules for $H$ are the same thing as the comodules for the quantum double $\mathscr{E}$. In particular, if $E$ denotes the largest (non-topological) subcoalgebra (which is a subbialgebra) of $\mathscr{E}$, then the category of right $E$-comodules is completely identified with the category of locally finite crossed $H$-bimodules.

The following remarks will be useful in later discussions.

3.3 Remark. The crossed axiom is enough to check for generators of the algebra $H$. 
In fact, the set of $h \in H$ satisfying the crossed axiom for all $v \in V$ forms a subalgebra.

Assume $H$ is a Hopf subalgebra of $U^{\circ}$ for some Hopf algebra $U$. For $x \in U$ and $h \in H$, define the actions [S, pp. 46, 100]

$$
x \rightarrow h=\sum h_{(1)}\left\langle x, h_{(2)}\right\rangle, \quad h \leftarrow x=\sum\left\langle x, h_{(1)}\right\rangle h_{(2)} .
$$

Recall [S, Sect. 2.1] that every right $H$-comodule has a natural left $U$-module structure. Hence, if $V$ is a right $H$-bimodule, we can think we have a bimodule ${ }_{U} V_{H}$ for the algebras $U, H$.

3.4 Remark. With the above assumptions, the crossed axiom is expressed

$$
\sum\left(x_{(1)} v\right)\left(x_{(2)} \rightarrow h\right)=\sum x_{(2)}\left(v\left(h<x_{(1)}\right)\right)
$$

for $v \in V, x \in U, h \in H$.

3.5 Remark. With the above assumptions, if the algebra $U$ is generated by a subcoalgebra $C$ and the algebra $H$ by a subset $\Lambda$, then the crossed axiom of 3.4 is enough to check for all $v \in V, x \in C$, and $h \in \Lambda$.

In fact, the set of $h \in H$ satisfying the crossed axiom in 3.4 for all $v \in V$ and $x \in C$ is a subalgebra, and the set of $x \in U$ satisfying it for all $v \in V$ and $h \in H$ is also a subalgebra.

\section{Quantum Lorentz Group}

We review the construction of the quantum Lorentz group $\operatorname{SL}_{q}(2, \mathbb{C})$ introduced by Podleś and Woronowicz [PW, Sects. 1,5].

Hereafter, we let $k=\mathbb{C}$ the complex numbers. A *-algebra means an algebra over $\mathbb{C}$ with an involutive conjugate linear automorphism $*$ such that $(a b)^{*}=b^{*} a^{*}$, $a, b \in A$. The tensor product of two *-algebras has a natural *-structure. A *-Hopf algebra means a Hopf algebra over $\mathbb{C}$ and a *-algebra $H$ such that the comultiplication $\Delta$ and the counit $\varepsilon$ are ${ }^{*}$-homomorphisms, i.e., $\Delta(h)^{*}=\Delta\left(h^{*}\right)$ and $\overline{\varepsilon(h)}=\varepsilon\left(h^{*}\right), h \in H$. If this is the case, the algebra opposite $H^{\text {op }}$ or the coalgebra opposite $H^{\text {cop }}$ is a Hopf algebra, too. This implies that the antipode $S$ of $H$ is bijective and one has

$$
* \circ=S^{-1 \circ *}, \text { or }\left(S_{\circ} *\right)^{2}=I .
$$

A topological *-algebra and a topological *-Hopf algebra are defined similarly.

If $H$ is a *-Hopf algebra, we give the following *-structure to the dual topological Hopf algebra $H^{\prime}$ :

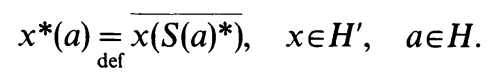

For a *-Hopf algebra $H$, let $\mathscr{E}=\operatorname{End}_{\mathbb{C}}(H)$ be the quantum double. As a topological algebra, this has a natural *-structure isomorphic to $H \widehat{\otimes} H^{\prime}$. If $f \in \mathscr{E}$, we have $f^{*}=* \circ f \circ * \circ S$, or

$$
f^{*}(a)=f\left(S(a)^{*}\right)^{*}, \quad a \in H .
$$


4.1 Proposition. $\mathscr{E}$ is a topological $*_{-}$Hopf algebra.

Proof. We show the comultiplication $\tilde{\Delta}$ commutes with $*$. If $f \in \mathscr{E}, \tilde{\Delta}(f)^{*}$ maps $x \otimes y$ in $H \otimes H$ to

which is

$$
\left[\tilde{\Delta}(f)\left(S(x)^{*} \otimes S(y)^{*}\right)\right]^{*}
$$

$$
\begin{gathered}
{\left[\sum\left(1 \otimes S\left(x_{(3)}\right)^{*}\right) \Delta\left(f\left(S(y)^{*} S\left(x_{(2)}\right)^{*}\right)\right)\left(1 \otimes x_{(1)}{ }^{*}\right)\right]^{*}} \\
\quad=\sum\left(1 \otimes x_{(1)}\right)\left[\Delta\left(f\left(S\left(y x_{(2)}\right)^{*}\right)\right)\right]^{*}\left(1 \otimes S\left(x_{(3)}\right)\right) .
\end{gathered}
$$

Since $\Delta$ and $*$ commute, it follows that $\tilde{\Delta}(f)^{*}=\tilde{\Delta}\left(f^{*}\right)$.

Note that the identity $I$ is unitary in the *algebra $\mathscr{E}$ in the sense that $I^{*}=I^{-1}(=S)$.

Fix a real parameter $q \neq 0, \pm 1$ in the rest of the paper. The following notations will be used throughout.

4.2 Definition. Let $B_{q}$ be the $\mathbb{C}$-algebra defined by generators $a, b, c, d$ and the following relations:

$$
\begin{aligned}
& b a=q a b, \quad c a=q a c, \quad d b=q b d, \quad d c=q c d, \\
& c b=b c, \quad a d-q^{-1} b c=1=d a-q b c .
\end{aligned}
$$

These relations tell that $\left(\begin{array}{cc}d & -q b \\ -q^{-1} c & a\end{array}\right)$ is the inverse of the matrix $\left(\begin{array}{ll}a & b \\ c & d\end{array}\right)$. The algebra $B_{q}$ has the following *-Hopf algebra structure:

$$
\begin{aligned}
\Delta\left(\begin{array}{ll}
a & b \\
c & d
\end{array}\right) & =\left(\begin{array}{ll}
a \otimes 1 & b \otimes 1 \\
c \otimes 1 & d \otimes 1
\end{array}\right)\left(\begin{array}{cc}
1 \otimes a & 1 \otimes b \\
1 \otimes c & 1 \otimes d
\end{array}\right), \\
\varepsilon\left(\begin{array}{ll}
a & b \\
c & d
\end{array}\right) & =\left(\begin{array}{ll}
1 & 0 \\
0 & 1
\end{array}\right), \\
\left(\begin{array}{ll}
a^{*} & c^{*} \\
b^{*} & d^{*}
\end{array}\right) & =S\left(\begin{array}{ll}
a & b \\
c & d
\end{array}\right)=\left(\begin{array}{cc}
d & -q b \\
-q^{-1} c & a
\end{array}\right) .
\end{aligned}
$$

The *-Hopf algebra $B_{q}$ corresponds to the compact quantum group $\mathrm{SU}_{q}(2)$ [W] [MMNNU].

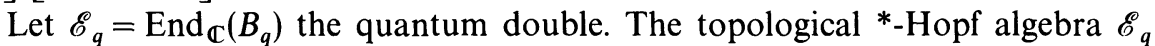
represents the double group of $\mathrm{SU}_{q}(2)$ [PW, Sect. 4]. The quantum Lorentz group $\mathrm{SL}_{q}(2, \mathbb{C})$ was introduced by Podleś and Woronowicz as a *-Hopf subalgebra of $\mathscr{E}_{q}$. We define a *-Hopf subalgebra $C_{q}$ of the topological dual $B_{q}^{\prime}$, and then a *-subalgebra $A_{q}$ of $B_{q} \otimes C_{q}$. We show $A_{q}$ is a Hopf subalgebra of $\mathscr{E}_{q}=B_{q} \hat{\otimes} B_{q}^{\prime}$. One sees later the *-Hopf algebra $A_{q}$ corresponds to the quantum Lorentz group $\mathrm{SL}_{q}(2, \mathbb{C})$.

Begin with two algebra maps $B_{q} \rightarrow \mathbb{C}$,

$$
\begin{aligned}
& p:\left(\begin{array}{ll}
a & b \\
c & d
\end{array}\right) \mapsto\left(\begin{array}{cc}
q^{1 / 2} & 0 \\
0 & q^{-1 / 2}
\end{array}\right), \\
& \tau:\left(\begin{array}{ll}
a & b \\
c & d
\end{array}\right) \mapsto\left(\begin{array}{rr}
-1 & 0 \\
0 & -1
\end{array}\right) .
\end{aligned}
$$


We think $p$ and $\tau$ are group-like elements on $B_{q}^{\prime}$. The actions $\tau \rightarrow, \leftarrow \tau$ on $B_{q}$ (see above 3.4) are the algebra automorphism

$$
\left(\begin{array}{ll}
a & b \\
c & d
\end{array}\right) \mapsto\left(\begin{array}{cc}
-a & -b \\
-c & -d
\end{array}\right)
$$

hence the group-like $\tau$ is central in $B_{q}^{\prime}$. We have

$$
\tau^{*}=\tau, \quad p^{*}=\left\{\begin{array}{lll}
p & \text { if } & q>0 \\
\tau p & \text { if } & q<0
\end{array}\right.
$$

in the *-algebra $B_{q}^{\prime}$. Here, and in the following, we note that the involution of $B_{q}$, $x \mapsto S(x)^{*}$, preserves $a$ and $d$, and exchanges $b$ and $c$.

For any $e \in \mathbb{C}$, there is an opposite algebra map $\pi_{e}: B_{q} \rightarrow M_{2}(\mathbb{C})$

$$
\begin{aligned}
& a \mapsto\left(\begin{array}{cc}
q^{1 / 2} & 0 \\
0 & q^{-1 / 2}
\end{array}\right), \quad b \mapsto\left(\begin{array}{ll}
0 & 0 \\
0 & 0
\end{array}\right), \\
& c \mapsto\left(\begin{array}{ll}
0 & e \\
0 & 0
\end{array}\right), \quad d \mapsto\left(\begin{array}{cc}
q^{-1 / 2} & 0 \\
0 & q^{1 / 2}
\end{array}\right) .
\end{aligned}
$$

Let us express

$$
\pi_{e}(x)=\left(\begin{array}{cc}
p(x) & n_{e}(x) \\
0 & p^{-1}(x)
\end{array}\right), x \in B_{q}
$$

with $n_{e} \in B_{q}^{\prime}$. The fact that $\pi_{e}$ is an opposite representation means we have in the topological Hopf algebra $B_{q}^{\prime}$,

$$
\Delta\left(n_{e}\right)=p^{-1} \otimes n_{e}+n_{e} \otimes p, \quad \varepsilon\left(n_{e}\right)=0 .
$$

In other words, $n_{e}$ is a $\left(p^{-1}, p\right)$-primitive.

4.4 Lemma. In the topological *-algebra $B_{q}^{\prime}$, we have

(1) $p^{*} p=p p^{*}$

(2) $n_{e} p=q p n_{e}$

(3) $n_{e} p^{*}=q p^{*} n_{e}$

(4) $n_{e}^{*} n_{e}-n_{e} n_{e}^{*}=\frac{e \bar{e}}{|q|\left(1-q^{-2}\right)}\left(p p^{*}-p^{-1}\left(p^{-1}\right)^{*}\right)$.

Proof. (1) follows from (4.3), and (3) from (2) plus (4.3). To prove (2) and (4), note that $p n_{e} p^{-1}$ is a $\left(p^{-1}, p\right)$-primitive and $\left[n_{e}^{*}, n_{e}\right]$ is a $\left(p^{-1}\left(p^{-1}\right)^{*}, p p^{*}\right)$-primitive. (The latter follows from the fact that $p^{-1} \otimes n_{e}$ and $n_{e}^{*} \otimes p^{*}$ commute. This fact is a consequence of (3).) In general, if $x, y$ are two $(g, \gamma)$-primitives in $B_{q}^{\prime}$ with group-likes $g, \gamma$, then a linear relation $y=\lambda x(\lambda \in \mathbb{C})$ will hold if it does on the generators $a, b, c, d$. The linear relation (2) becomes at the generating matrix $\left(\begin{array}{ll}a & b \\ c & d\end{array}\right)$,

$$
\left(\begin{array}{ll}
0 & 0 \\
e & 0
\end{array}\right)\left(\begin{array}{cc}
q^{1 / 2} & 0 \\
0 & q^{-1 / 2}
\end{array}\right)=q\left(\begin{array}{cc}
q^{1 / 2} & 0 \\
0 & q^{-1 / 2}
\end{array}\right)\left(\begin{array}{ll}
0 & 0 \\
e & 0
\end{array}\right),
$$

which is true. Similarly, one can check (4). 
Let $n=n_{e}$ for $e=q^{1 / 2}\left(1-q^{-2}\right)$ so that we have

$$
\left[n^{*}, n\right]=\left(1-q^{-2}\right)\left(p p^{*}-p^{-1}\left(p^{-1}\right)^{*}\right) \text {. }
$$

4.5 Definition. Let $C_{q}$ be the *-subalgebra of $B_{q}^{\prime}$ generated by $p, p^{-1}$, and $n$.

It is a *-Hopf subalgebra. It will be natural to consider $C_{q}$ as a ${ }^{*}$-Hopf subalgebra of $B_{q}^{\prime \text { cop }}$ (but not of $B_{q}^{\prime}$ ), since we started with anti-representation $\pi_{e}$.

We have the following inclusions of *-algebras:

$$
B_{q} \otimes C_{q} \subset B_{q} \otimes B_{q}^{\prime} \subset B_{q} \hat{\otimes} B_{q}^{\prime}=\mathscr{E}_{q} .
$$

4.6 Definition. Let $A_{q}$ be the *-subalgebra of $B_{q} \otimes C_{q}$ generated by the elements $\alpha, \beta, \gamma, \delta$ defined as follows:

$$
\left(\begin{array}{ll}
\alpha & \beta \\
\gamma & \delta
\end{array}\right)=\left(\begin{array}{ll}
a & b \\
c & d
\end{array}\right)\left(\begin{array}{cc}
p & n \\
0 & p^{-1}
\end{array}\right)
$$

Note that we have

$$
\left(\begin{array}{ll}
\alpha^{*} & \gamma^{*} \\
\beta^{*} & \delta^{*}
\end{array}\right)=\left(\begin{array}{cc}
p^{*} & 0 \\
n^{*} & \left(p^{-1}\right)^{*}
\end{array}\right)\left(\begin{array}{cc}
d & -q b \\
-q^{-1} c & a
\end{array}\right) .
$$

4.7 Proposition. The following 17 relations hold among $\alpha, \beta, \gamma, \delta$ :
(1) $\beta \alpha=q \alpha \beta$,
(2) $\gamma \alpha=q \alpha \gamma$,
(3) $\delta \beta=q \beta \delta$,
(4) $\delta \gamma=q \gamma \delta$,

(5) $\gamma \beta=\beta \gamma$,

(6) $\delta \alpha-q \beta \gamma=1$

(7) $\alpha \delta-q^{-1} \beta \gamma=1$,

(8) $\alpha^{*} \delta=\delta \alpha^{*}$,

(9) $\beta^{*} \gamma=\gamma \beta^{*}$

(10) $\gamma^{*} \gamma=\gamma \gamma^{*}$

(11) $\alpha^{*} \gamma=q \gamma \alpha^{*}$,

(12) $\gamma^{*} \delta=q^{-1} \delta \gamma^{*}$

(13) $\alpha^{*} \alpha=\alpha \alpha^{*}+\left(q^{-2}-1\right) \gamma \gamma^{*}, \quad$ (14) $\delta^{*} \delta=\delta \delta^{*}+\left(1-q^{-2}\right) \gamma \gamma^{*}$,

(15) $\alpha^{*} \beta=q^{-1} \beta \alpha^{*}+q^{-1}\left(q^{-2}-1\right) \delta \gamma^{*}$,

(16) $\beta^{*} \delta=q \delta \beta^{*}+q\left(1-q^{-2}\right) \gamma \alpha^{*}$,

(17) $\beta^{*} \beta=\beta \beta^{*}+\left(1-q^{-2}\right)\left(\alpha \alpha^{*}-\delta \delta^{*}\right)-\left(1-q^{-2}\right)^{2} \gamma \gamma^{*}$.

Proof. By using the previous Lemma 4.4, one checks that $a, b, c, d$ and $p, n, 0, p^{-1}$ satisfy the 17 relations. Since they commute "doubly," the claim will follow from [PW, Proposition 1.1, p. 389].

4.8 Proposition. We have

$$
\tilde{\Delta}\left(\begin{array}{ll}
\alpha & \beta \\
\gamma & \delta
\end{array}\right)=\left(\begin{array}{ll}
\alpha \otimes 1 & \beta \otimes 1 \\
\gamma \otimes 1 & \delta \otimes 1
\end{array}\right)\left(\begin{array}{cc}
1 \otimes \alpha & 1 \otimes \beta \\
1 \otimes \gamma & 1 \otimes \delta
\end{array}\right), \quad \tilde{\varepsilon}\left(\begin{array}{ll}
\alpha & \beta \\
\gamma & \delta
\end{array}\right)=\left(\begin{array}{ll}
1 & 0 \\
0 & 1
\end{array}\right),
$$

where $\tilde{\Delta}, \tilde{\varepsilon}$ the coalgebra structures of $\mathscr{E}_{q}$.

Proof. Define the following coaction on a 2 -dimensional vector space $\mathbb{C} u \oplus \mathbb{C} v$

$$
u \mapsto u \otimes \alpha+v \otimes \gamma, \quad v \mapsto u \otimes \beta+v \otimes \delta .
$$

The statement means that this satisfies the comodule condition for $\mathscr{E}_{q}$. In fact, this is precisely the $\mathscr{E}_{q}$-comodule corresponding to the 2-dimensional crossed $B_{q}$-bimodule $V_{1}(5.2)$. 
If $\tilde{S}$ denotes the antipode of $\mathscr{E}_{q}$, we have

$$
\tilde{S}\left(\begin{array}{ll}
\alpha & \beta \\
\gamma & \delta
\end{array}\right)=\left(\begin{array}{ll}
\alpha & \beta \\
\gamma & \delta
\end{array}\right)^{-1}=\left(\begin{array}{cc}
\delta & -q \beta \\
-q^{-1} \gamma & \alpha
\end{array}\right) .
$$

It follows that $A_{q}$ is a ${ }^{*}$-Hopf subalgebra of $\mathscr{E}_{q}$.

Let $\tilde{\tau}=1 \otimes \tau$ which is a central group-like element of order 2 in $B_{q} \widehat{\otimes} B_{q}^{\prime}=\mathscr{E}_{q}$. The following theorem is the main result of the paper.

4.9 Theorem. (a) The *-algebra $A_{q}$ is defined by generators $\alpha, \beta, \gamma, \delta$ and the 17 relations of 4.7 .

(b) We have $A_{q}+A_{q} \tilde{\tau}=A_{q} \oplus A_{q} \tilde{\tau}$.

(c) $A_{q} \oplus A_{q} \tilde{\tau}$ coincides with the largest subcoalgebra of the quantum double $\mathscr{E}_{q}=\operatorname{End}_{\mathbb{C}}\left(B_{q}\right)$.

In the next section, the statement will be reduced to some representationtheoretic facts.

(a) means the *-Hopf algebra $A_{q}$ corresponds to the quantum Lorentz group $\mathrm{SL}_{q}(2, \mathbb{C})$. One will see (c) answers the conjecture of Podleś and Woronowicz [PW, 6.4].

\section{Crossed $B_{q}$-Bimodules}

We recall the embedding of $B_{q}$ into a Hopf subalgebra of $U_{q}^{\circ}$, where $U_{q}=\mathrm{U}_{q}(\mathfrak{s l}(2))$ [T2], and express the crossed axiom in the form of 3.4 explicitly.

Let $U_{q}$ be the $\mathbb{C}$-algebra defined by generators $K, K^{-1}, E, F$ and the relations

$$
\begin{aligned}
K K^{-1} & =1=K^{-1} K, \\
K E K^{-1} & =q^{2} E, \quad K F K^{-1}=q^{-2} F, \\
{[E, F] } & =\frac{K-K^{-1}}{q-q^{-1}} .
\end{aligned}
$$

We give it the following Hopf algebra structure:

$$
\begin{aligned}
& \Delta(K)=K \otimes K, \quad \varepsilon(K)=1, \quad S(K)=K^{-1}, \\
& \Delta(E)=1 \otimes E+E \otimes K, \quad \varepsilon(E)=0, \quad S(E)=-E K^{-1}, \\
& \Delta(F)=K^{-1} \otimes F+F \otimes 1, \quad \varepsilon(F)=0, \quad S(F)=-K F .
\end{aligned}
$$

We can embed the Hopf algebra $B_{q}(4.2)$ into $U_{q}^{\circ}$ as follows. Let $\lambda: U_{q} \rightarrow \mathrm{M}_{2}(\mathbb{C})$ be the basic representation

$$
K \mapsto\left(\begin{array}{cc}
q & 0 \\
0 & q^{-1}
\end{array}\right), \quad E \mapsto\left(\begin{array}{ll}
0 & 1 \\
0 & 0
\end{array}\right), \quad F \mapsto\left(\begin{array}{ll}
0 & 0 \\
1 & 0
\end{array}\right)
$$

and write

$$
\lambda(x)=\left(\begin{array}{cc}
\tilde{a}(x) & \tilde{b}(x) \\
\tilde{c}(x) & \tilde{d}(x)
\end{array}\right), \quad x \in U_{q}
$$


with $\tilde{a}, \tilde{b}, \tilde{c}, \tilde{d} \in U_{q}^{\circ}$. Then, $\left(\begin{array}{ll}a & b \\ c & d\end{array}\right) \mapsto\left(\begin{array}{ll}\tilde{a} & \tilde{b} \\ \tilde{c} & \tilde{d}\end{array}\right)$ induces an injective Hopf algebra $\operatorname{map} B_{q} \rightarrow U_{q}^{\circ}$, and more precisely, we have $U_{q}^{\circ}=B_{q} \oplus B_{q} \gamma$, where $\gamma: B_{q} \mapsto \mathbb{C}$ the algebra map $K \mapsto-1, E, F \mapsto 0$ (see [T2, 3.11]).

The actions $\rightarrow, \leftarrow$ (above 3.4) have the following descriptions:

$$
\begin{aligned}
K \rightarrow\left(\begin{array}{ll}
a & b \\
c & d
\end{array}\right) & =\left(\begin{array}{ll}
q a & q^{-1} b \\
q c & q^{-1} d
\end{array}\right), \quad\left(\begin{array}{ll}
a & b \\
c & d
\end{array}\right)<K=\left(\begin{array}{cc}
q a & q b \\
q^{-1} c & q^{-1} d
\end{array}\right), \\
E \rightarrow\left(\begin{array}{ll}
a & b \\
c & d
\end{array}\right) & =\left(\begin{array}{ll}
0 & a \\
0 & c
\end{array}\right), \quad\left(\begin{array}{ll}
a & b \\
c & d
\end{array}\right)-E=\left(\begin{array}{ll}
c & d \\
0 & 0
\end{array}\right), \\
F \rightarrow\left(\begin{array}{ll}
a & b \\
c & d
\end{array}\right) & =\left(\begin{array}{ll}
b & 0 \\
d & 0
\end{array}\right), \quad\left(\begin{array}{ll}
a & b \\
c & d
\end{array}\right) \leftarrow F=\left(\begin{array}{ll}
0 & 0 \\
a & b
\end{array}\right) .
\end{aligned}
$$

It follows from 3.5 Remark that the crossed $B_{q}$-bimodules admit the following description.

5.1 Proposition. Let $V$ be a right $B_{q^{-}}$-bimodule. If we consider $V$ as a left $U_{q^{-}}$and a right $B_{q}$-module, then the crossed axiom is equivalent to the following 12 commutation relations.

(1) $K(v a)=(K v) a, \quad(2) K(v b)=q^{-2}(K v) b$,

(3) $K(v c)=q^{2}(K v) c$, (4) $K(v d)=(K v) d$,

(5) $E(v a)=q(E v) a-q^{2}(K v) c$, (6) $E(v b)=q^{-1}(E v) b+v a-(K v) d$,

(7) $E(v c)=q(E v) c$, (8) $E(v d)=q^{-1}(E v) d+v c$,

(9) $F(v a)=q(F v) a+q\left(K^{-1} v\right) b, \quad(10) F(v b)=q(F v) b$,

(11) $F(v c)=q^{-1}(F v) c+q^{-1}\left(K^{-1} v\right) d-q^{-1} v a$, (12) $F(v d)=q^{-1}(F v) d-q^{-1} v b$, where $v \in V$.

5.2 Example. Let $V_{1}=\mathbb{C} u+\mathbb{C} v$ a 2-dimensional vector space. Give the following $B_{q}$-bimodule structure on $V_{1}$.

$$
\begin{array}{ll}
\text { comodule } & \left\{\begin{array}{l}
u \mapsto u \otimes a+v \otimes c, \\
\text { structure } \\
v \mapsto u \otimes b+v \otimes d,
\end{array}\right. \\
\text { module } & \left\{\begin{array}{l}
u x=p(x) u, \\
\text { structure }
\end{array} \quad x \in B_{q} .\right.
\end{array}
$$

The corresponding actions of $K, E, F$ and $a, b, c, d$ are as follows:

$$
\begin{aligned}
& K u=q u, \quad K v=q^{-1} v, \quad E u=0, \quad E v=u, \quad F u=v, \quad F v=0, \\
& u a=q^{1 / 2} u, \quad v a=q^{-1 / 2} v, \quad u b=0, \quad v b=0, \\
& u c=0, \quad v c=q^{1 / 2}\left(1-q^{-2}\right) u, \quad u d=q^{-1 / 2} u, \quad v d=q^{1 / 2} v .
\end{aligned}
$$

One verifies the 12 commutation relations hold. Hence $V_{1}$ is a crossed $B_{q}$-bimodule. (Note that the choice $e=q^{1 / 2}\left(1-q^{-2}\right)$ is compulsory.) 
All finite dimensional $U_{q}$-modules are completely reducible, and for each integer $k \geqq 0$, there are two simple $U_{q}$-modules of dimension $k+1$. One is a comodule for $B_{q}$ and the other for $B_{q} \gamma$. Let $V_{k}$ be the simple $B_{q}$-comodule of dimension $k+1$. It has the highest weight $q^{k}$ and the lowest weight $q^{-k}$. Every $B_{q}$-comodule is the direct sum of a set of $V_{k}, k \in \mathbb{N}$ [T2, Sects. 2, 3].

Let $B_{q}^{\text {op }}$ the algebra opposite of $B_{q}$ with the same coalgebra structure. It is identified with $B_{q^{-1}}$. The maps $\left(\begin{array}{ll}a & b \\ c & d\end{array}\right) \mapsto\left(\begin{array}{ll}\alpha & \beta \\ \gamma & \delta\end{array}\right),\left(\begin{array}{cc}\alpha^{*} & \beta^{*} \\ \gamma^{*} & \delta^{*}\end{array}\right)$ induce Hopf algebra maps

$$
B_{q} \rightarrow A_{q} \quad \text { and } \quad B_{q}^{\mathrm{op}} \rightarrow A_{q}
$$

Let $\Phi$ be the composite

$$
B_{q} \otimes B_{q}^{\text {op }} \rightarrow A_{q} \otimes A_{q} \stackrel{\text { mult }}{\longrightarrow} A_{q},
$$

which is a coalgebra map.

Let $V_{k}^{*}$ be the simple $B_{q}^{\text {op }}$-comodule of dimension $k+1$. Then $V_{k} \otimes V_{l}^{*},(k, l) \in \mathbb{N}^{2}$ give a complete set of representatives for the isomorphism classes of all simple comodules for $B_{q} \otimes B_{q}^{\text {op }}$.

Every comodule for $B_{q} \otimes B_{q}^{\text {op }}$ has an $A_{q}$-comodule structure through $\Phi$, hence the structure of a crossed $B_{q}$-bimodule.

5.3 Definition. For integers $k, l \geqq 0$, let $V_{k, l}$ denote the $A_{q}$-comodule $V_{k} \otimes V_{l}^{*}$. It is also considered as a crossed $B_{q}$-bimodule.

An explicit description of the actions of $K, E, F$ and $a, b, c, d$ on $V_{k, l}$ will be given in the next section (6.4). One sees 5.2 Example gives a description of $V_{1,0}$.

When $V$ is a crossed $B_{q}$-bimodule, let $V^{(-)}$denote the $B_{q}$-comodule $V$ on which $a, b, c, d$ act by the operations of $-a,-b,-c,-d$. One verifies the 12 commutation relations for $V^{(-)}$. The corresponding comodule structure for the quantum double $\mathscr{E}_{q}=\operatorname{End}_{\mathbb{C}}\left(B_{q}\right)$ is obtained through the multiplication of $\tilde{\tau}$.

The following two theorems will be proved in the next two sections.

5.4 Theorem. $V_{k, l}$ and $V_{k, l}^{(-)}$give a complete set of representatives for the isomorphism classes of all simple finite dimensional crossed $B_{q}$-bimodules.

5.5 Theorem. All finite dimensional crossed $B_{q}$-bimodules are completely reducible.

We deduce 4.9 Theorem from these results. Let $\tilde{A}_{q}$ be the *-algebra defined by generators $\alpha, \beta, \gamma, \delta$ and the 17 relations of 4.7. It has a $*$-Hopf algebra structure given by 4.8 , and it corresponds to the quantum Lorentz group $\operatorname{SL}_{q}(2, \mathbb{C})$. There is a canonical surjective ${ }^{*}$-Hopf algebra map $\widetilde{A}_{q} \rightarrow A_{q}$, and the coalgebra map $\Phi$ factors as

$$
\Phi: B_{q} \otimes B_{q}^{\mathrm{op}} \rightarrow \tilde{A}_{q} \rightarrow A_{q} .
$$

Let $E_{q}$ be the largest subcoalgebra of $\mathscr{E}_{q}=\operatorname{End}_{\mathbb{C}}\left(B_{q}\right) .5 .5$ Theorem means $E_{q}$ is cosemisimple, and 5.4 Theorem means it is the direct sum of the coefficient spaces of simple comodules $V_{k, l}$ and $V_{k, l}^{(-)}, k, l \in \mathbb{N}$. In other words, the map

$$
\Phi+\Phi \tilde{\tau}:\left(B_{q} \otimes B_{q}^{\mathrm{op}}\right) \oplus\left(B_{q} \otimes B_{q}^{\mathrm{op}}\right) \rightarrow A_{q}+A_{q} \tilde{\tau} \subset \mathscr{E}_{q}
$$

is injective and has image $E_{q}$. Since $A_{q}+A_{q} \tilde{\tau}$ is contained in $E_{q}$, it follows that 
$E_{q}=A_{q}+A_{q} \tilde{\tau}=A_{q} \oplus A_{q} \tilde{\tau}$ and that $\Phi$ is an isomorphism. This implies the projection $\tilde{A}_{q} \rightarrow A_{q}$ is an isomorphism, and the statements (a), (b), (c) of 4.9 follow.

As a corollary of 5.4 and 5.5 , it follows that every locally finite crossed $B_{q}$-bimodule (or a locally finite representation of $\mathrm{SL}_{q}(2, \mathbb{C})$ in the terminology of [PW]) $V$ is isomorphic to the direct sum $V_{1} \oplus V_{2}^{(-)}$for some uniquely determined (up to isomorphisms) $A_{q}$-comodules $V_{1}, V_{2} . A_{q}$-comodules are smooth representations of $\mathrm{SL}_{q}(2, \mathbb{C})$ in the terminology of $[\mathrm{PW}]$. Thus our results give an affirmative answer to the Conjecture 6.4 of [PW].

\section{Finite Dimensional Simple Crossed $\boldsymbol{B}_{q}$-Bimodules}

We prove 5.4 Theorem. We begin with describing the bimodule structure of $V_{k, l}$. The bimodule structure of $V_{1}=\mathbb{C} u \oplus \mathbb{C} v$ is described in 5.2. We can identify $V_{k}$ as the subspace of $V_{1} \otimes \cdots \otimes V_{1}$ ( $k$ copies spanned by $F^{i} v_{k}$. $0 \leqq i \leqq k$, with $v_{k}=u \otimes \cdots \otimes u$. It is a sub-bimodule, and the following description follows easily by induction using the 12 commutation relations of 5.1 ,

$$
\begin{aligned}
& \left(F^{i} v_{k}\right) a=q^{(k / 2)-i} F^{i} v_{k}, \quad\left(F^{i} v_{k}\right) b=0, \\
& \left(F^{i} v_{k}\right) c=q^{-(k / 2)+i}\left(1-q^{-2}\right)[i][k+1-i] F^{i-1} v_{k}, \quad\left(F^{i} v_{k}\right) d=q^{-(k / 2)+i} F^{i} v_{k},
\end{aligned}
$$

for $0 \leqq i \leqq k$. Here and in the following, we use the notation

$$
[i]=\frac{q^{i}-q^{-i}}{q-q^{-1}} .
$$

The expression $\left(F^{i} v_{k}\right) c$ should be interpreted to mean 0 when $i=0$, since $[0]=0$ (though $F^{-1} v_{k}$ has no meaning). Such a convention will be used in the following.

The description of $V_{1}^{*}\left(=V_{0,1}\right)$ involves the signature of $q$. Let $\eta$ be the signature of $q$ in the rest of the paper. By definition, the $A_{q}$-comodule $V_{1}^{*}$ has a base $u^{*}, v^{*}$ such that the coaction is given by

$$
v^{*} \mapsto v^{*} \otimes \alpha^{*}+u^{*} \otimes \gamma^{*}, \quad u^{*} \mapsto v^{*} \otimes \beta^{*}+u^{*} \otimes \delta^{*} .
$$

By using the expression below 4.6, one sees the actions of $K, E, F$ and $a, b, c, d$ on $V_{1}^{*}$ are described as follows.

$$
\begin{aligned}
& K u^{*}=q u^{*}, \quad K v^{*}=q^{-1} v^{*}, \quad E u^{*}=0, \quad E v^{*}=-q u^{*} \\
& F u^{*}=-q^{-1} v^{*}, \quad F v^{*}=0, \\
& u^{*} a=\eta q^{-1 / 2} u^{*}, \quad v^{*} a=\eta q^{1 / 2} v^{*}, \quad u^{*} b=\eta q^{1 / 2}\left(1-q^{-2}\right) v^{*}, \quad v^{*} b=0 \\
& u^{*} c=0, \quad v^{*} c=0, \quad u^{*} d=\eta q^{1 / 2} u^{*}, \quad v^{*} d=\eta q^{-1 / 2} v^{*} .
\end{aligned}
$$

We can identify $V_{l}^{*}$ as the span of $E^{i} v_{-l}, 0 \leqq i \leqq l$ in $V_{1}^{*} \otimes \cdots \otimes V_{1}^{*}(l$ copies $)$, where $v_{-l}=v^{*} \otimes \cdots \otimes v^{*}$. Similarly as before, one verifies the following expressions:

$$
\begin{aligned}
& \left(E^{i} v_{-l}\right) a=\eta^{l} q^{(l / 2)-i} E^{i} v_{-l}, \\
& \left(E^{i} v_{-l}\right) b=-\eta^{l} q^{1-(l / 2)+i}\left(1-q^{-2}\right)[i][l+1-i] E^{i-1} v, \\
& \left(E^{i} v_{-l}\right) c=0, \quad\left(E^{i} v_{-l}\right) d=\eta^{l} q^{-(l / 2)+i} E^{i} v_{-l}
\end{aligned}
$$


It follows from (6.1) and (6.3) that the crossed bimodule $V_{k . l}$ has a base $v_{i, j}=F^{i} v_{k} \otimes E^{j} v_{-l}, 0 \leqq i \leqq k, 0 \leqq j \leqq l$, and admits the following description of the actions:

$$
\begin{aligned}
v_{i, j} a & =\eta^{l} q^{((k+l) / 2)-i-j} v_{i, j}, \\
v_{i, j} b & =-\eta^{l} q^{1+((k-l) / 2)+j-1}\left(1-q^{-2}\right)[j][l+1-j] v_{i, j-1}, \\
v_{i, j} c & =\eta^{l} q^{((l-k) / 2)+i-j}\left(1-q^{-2}\right)[i][k+1-i] v_{i-1, j}, \\
v_{i, j} & =\eta^{l} q^{-((k+l) / 2)+i+j}\left(v_{i, j}-q\left(1-q^{-2}\right)^{2}[i][j][k+1-i][l+1-j] v_{i-1, j-1}\right), \\
K v_{i, j} & =q^{k-l+2(j-i)} v_{i, j}, \\
E v_{i, j} & =v_{i, j+1}+q^{2 j-l}[i][k-i+1] v_{i-1, j}, \\
F v_{i, j} & =v_{i+1, j}+q^{2 i-k}[j][l-j+1] v_{i, j-1} .
\end{aligned}
$$

Here, we use the convention that $v_{i, j}$ means 0 unless $0 \leqq i \leqq k$ or $0 \leqq j \leqq l$.

The description (6.4) tells that the following modified crossed bimodules are more natural objects to study.

6.5 Definition. For integers $k, l \geqq 0$, let $\tilde{V}_{k, l}$ mean $V_{k, l}$ if $q>0$ or $l$ even, and $V_{k, l}^{(-)}$ otherwise.

The crossed bimodule $\tilde{V}_{k, l}$ has an expression which is obtained from (6.4) by omitting the factor $\eta^{l}$.

6.6 Corollary. If we put $\tilde{v}=v_{k, l}$ in $\tilde{V}_{k, l}$, then we have

$$
\tilde{v} a=q^{-(k+l) / 2} \tilde{v}, \quad \tilde{v} b^{l+1}=0=\tilde{v} c^{k+1},
$$

and $\tilde{v} b^{i} c^{j}, 0 \leqq i \leqq l, 0 \leqq j \leqq k$, form a base for $\tilde{V}_{k, l}$. We have

$$
\begin{aligned}
K\left(\tilde{v} b^{i} c^{j}\right)= & q^{l-k+2(j-i)} \tilde{v} b^{i} c^{j} \\
E\left(\tilde{v} b^{i} c^{j}\right)= & -q^{2+((l-k) / 2)+j-i}\left(1-q^{-2}\right)[i][l+1-i] \tilde{v} b^{i-1} c^{j} \\
& +\frac{q^{-(k / 2)+(3 / 2) l-3 i+j}}{1-q^{-2}} \tilde{v} b^{i} c^{j+1}, \\
E\left(\tilde{v} b^{i} c^{j}\right)= & q^{1+((k-l) / 2)+i-j}\left(1-q^{-2}\right)[j][k+1-j] \tilde{v} b^{i} c^{j-1} \\
& -\frac{q^{-1-(l / 2)+(3 / 2) k-3 j+i}}{1-q^{-2}} \tilde{v} b^{i+1} c^{j}
\end{aligned}
$$

6.7 Proposition. The crossed $B_{q}$-bimodules $V_{k, l}$ and $V_{k, l}^{(-)}$are simple and pairwise non-isomorphic.

Proof. We show $\tilde{V}_{k, l}$ is simple. The base element $v_{i, j}$ has weight $k-l+2(j-i)$ relative to $K$ and weight $\frac{k+l}{2}-i-j$ relative to $a$. The pairs of weights are distinct for distinct $(i, j)$. Hence a subbimodule of $\tilde{V}_{k, l}$ is spanned by a set of $v_{i, j}$. In particular, it contains some $v_{i, j}$ if it is non-zero. Applying $b$ and $c$, one sees it contains $v_{0,0}$. It is easy to see $\widetilde{V}_{k, l}^{i, j}$ is generated by $v_{0,0}$ over $U_{q}$. This implies $\widetilde{V}_{k, l}$ (and $\widetilde{V}_{k, l}^{(-)}$also) is simple. To see they are mutually non-isomorphic, note that $\widetilde{V}_{k, l}$ has highest 
weight $k+l$ relative to $K$, and the corresponding weight vector has weight $\frac{k-l}{2}$ relative to $a$. Since the pair $\left(k+l, \frac{k-l}{2}\right)$ determines $(k, l)$, it follows that $V_{k, l}$ and $V_{k, l}^{(-)}$for all $k, l$ are mutually non-isomorphic.

For a crossed $B_{q}$-bimodule $V$ and $\lambda, \mu \in \mathbb{C}$, let $V_{\lambda, \mu}$ be the subspace of $v \in V$ such that $K v=\lambda v$ and $v a=\mu v$. It follows from the commutation relations of $b, c$ with $K, a$ (4.2 and 5.1), that we have

$$
V_{\lambda, \mu} b \subset V_{q^{-2} \lambda, q \mu}, \quad V_{\lambda, \mu} c \subset V_{q^{2} \lambda, q \mu} .
$$

6.8 Lemma. If $V$ is finite dimensional, $V_{\lambda, 0}=0$.

Proof. We may assume $\lambda \neq 0$. Since $V_{\lambda, 0} b \subset V_{q^{-2} \lambda, 0}, b$ is nilpotent on $V_{\lambda, 0}$. Similarly, $c$ is nilpotent, too. Since $1=a d-q^{-1} b c$, we have $v=-q^{-1} v b c$ for $v \in V_{\lambda, 0}$. This implies $v=0$,

6.9 Lemma. Let $v \in V_{\lambda, \mu}$. We have

(1) $v c-\left(1-q^{-2}\right) \lambda^{-1} \mu E v \in V_{q^{2} \lambda, q^{-1} \mu}$,

(2) $v b+q\left(1-q^{-2}\right) \lambda \mu F v \in V_{q^{-2} \lambda, q^{-1} \mu}$.

Proof. (1) Let $w$ be the element of the left-hand side. Since

$$
(E v) a=q^{-1} E(v a)+q^{-1} K(v c)=q^{-1} \mu E v+q \lambda v c,
$$

we have

$$
\begin{aligned}
w a & =q \mu v c-\left(1-q^{-2}\right) \lambda^{-1} \mu\left(q^{-1} \mu E v+q \lambda v c\right) \\
& =q^{-1} \mu v c-q^{-1}\left(1-q^{-2}\right) \lambda^{-1} \mu^{2} E v=q^{-1} \mu w .
\end{aligned}
$$

Since $v c$ and $E v$ have weight $q^{2} \lambda$ relative to $K$, so is $w$. (2) is similar.

Let $V$ be a finite dimensional simple crossed $B_{q}$-bimodule. We show $V$ is isomorphic to $V_{k, l}$ or $V_{k, l}^{(-)}$for some natural numbers $k, l$.

There are $\lambda, \mu \in \mathbb{C}-\{0\}$ such that $V_{\lambda, \mu} \neq 0$, since the actions of $K$ and $a$ commute with each other. We can choose $\lambda, \mu$ so that $V_{v, q^{-s}}=0$ for all $v \in \mathbb{C}$ and integers $s>0$. Take a non-zero element $v$ in $V_{\lambda, \mu}$. Since $V_{q^{2} \lambda, q^{-1} \mu}=0=V_{q^{-2} \lambda, q^{-1} \mu}$, it follows from 6.9 that

$$
v b=-q\left(1-q^{-2}\right) \lambda \mu F v \quad \text { and } \quad v c=\left(1-q^{-2}\right) \lambda^{-1} \mu E v .
$$

Note that $v b^{i} c^{j}$ is in $V_{q^{2(j-i)} \lambda, q^{i+j} \mu}$. Hence they are linearly independent if non-zero.

6.11 Lemma. $v b^{i} c^{j} d=\mu^{-1} q^{-i-j}\left(v b^{i} c^{j}+q^{-1} v b^{i+1} c^{j+1}\right)$.

Proof. Apply $1=a d-q^{-1} b c$ to $v b^{i} c^{j}$.

6.12 Lemma. We have

(1) $E\left(v b^{i}\right)=\left(\mu-\mu^{-1} \lambda q^{2(1-i)}\right)[i] v b^{i-1}+\lambda \mu^{-1} q^{-3 i}\left(1-q^{-2}\right)^{-1} v b^{i} c$,

(2) $F\left(v c^{j}\right)=-q^{-1}\left(\mu-\lambda^{-1} \mu^{-1} q^{2(1-j)}\right)[j] v c^{j-1}-\lambda^{-1} \mu^{-1} q^{-1-3 j}\left(1-q^{-2}\right)^{-1} v b c^{j}$.

Proof. (1) If $i=0$, this is (6.10). (We use the convention that [0]vb $b^{-1}$ means 0 ). 
We have by (6) of 5.1,

$$
E\left(v b^{i}\right)=q^{-1}\left(E\left(v b^{i-1}\right)\right) b+v b^{i-1} a-K\left(v b^{i-1} d\right),
$$

where $v b^{i-1} a=q^{i-1} \mu v b^{i-1}$ and $v b^{i-1} d=\mu^{-1} q^{1-i}\left(v b^{i-1}+q^{-1} v b^{i} c\right)$ by 6.11 . Hence $K\left(v b^{i-1} d\right)=\mu^{-1} q^{3(1-i)} \lambda\left(v b^{i-1}+q^{-1} v b^{i} c\right)$. Using this, the claim follows by induction. (2) is similar.

There are only finitely many pairs of integers $(i, j)$ such that $v b^{i} c^{j} \neq 0$, since they are linearly independent. Take the largest integers $k, l \geqq 0$ such that $v c^{k} \neq 0$ and $v b^{l} \neq 0$. It follows from (6.10) and (7) (respectively (10)) of 5.1 that the vector $E^{j} v\left(\right.$ respectively $F^{i} v$ ) is proportional to $v c^{j}$ (respectively $v b^{i}$ ). Hence,

$$
E^{k} v \neq 0, \quad E^{k+1} v=0 ; \quad F^{l} v \neq 0, \quad F^{l+1} v=0 .
$$

It follows from [APW, 1.11 Lemma] that

$$
\lambda=q^{l-k} \text {. }
$$

Letting $i=l+1$ in (1) of 6.12 , we conclude that

$$
\mu^{2}=\lambda q^{-2 l}=q^{-k-l} \text {, so } \mu= \pm q^{-(k+l) / 2} \text {. }
$$

Assume we have $\mu=q^{-(k+l) / 2}$.

6.13 Proposition. The correspondence $\tilde{v} b^{i} c^{j} \mapsto v b^{i} c^{j}$ gives an isomorphism of $B_{q}$-bimodules $\widetilde{V}_{k, l} \cong V$.

Proof. Call the above correspondence $\phi: \tilde{V}_{k, l} \rightarrow V$. This map commutes with the actions of $K$ and $a$, since $\tilde{v}$ and $v$ have the same weights relative to $K$ and $a$. Obviously, it commutes with the actions of $b, c$. By using 6.12 Lemma and the fact that

$$
E\left(v b^{i} c^{j}\right)=q^{j}\left(E\left(v b^{i}\right)\right) c^{j}, \quad F\left(v b^{i} c^{j}\right)=q^{i}\left(F\left(v c^{j}\right)\right) b^{i},
$$

one sees easily that the last two identities of 6.6 Corollary hold with $\tilde{v}$ replaced by $v$. Thus, $\phi$ commutes with $a, b, c$ and $K, E, F$. It commutes with $d$, too, since 6.11 Lemma (with $\mu=q^{-(k+l) / 2}$ ) holds for $\tilde{v}$. It follows that $\phi$ is a homomorphism of $B_{q}$-bimodules. Since both $\tilde{V}_{k, l}$ and $V$ are simple crossed bimodules, one concludes that $\phi$ is an isomorphism.

In case $\mu=-q^{-(k+l) / 2}$, one gets an isomorphism $\tilde{V}_{k, l}^{(-)} \cong V$. This concludes the proof of 5.4 Theorem.

\section{Complete Reducibility}

We prove 5.5 Theorem.

A Hopf algebra $H$ over a general field $k$ is called co-semisimple if all (right or left) $H$-comodules are completely reducible [S, XIV]. The following criterion seems fairly well-known among specialists, but it is difficult to find an explicit literature.

7.1 Proposition. The following are equivalent.

(1) The Hopf algebra $H$ is cosemisimple.

(2) All exact sequences of $H$-comodules of the form

$$
0 \rightarrow W \rightarrow V \rightarrow k \rightarrow 0
$$


split, where $k$ denotes the trivial 1-dimensional comodule.

(3) (2) is true for all simple comodules $W$.

Proof. (3) $\Rightarrow(2)$ is an easy exercise. To prove (2) $\Rightarrow(1)$, let $V$ be a comodule for $H$ and $W$ a subcomodule of $V$. We show $W$ is a direct summand of $V$. We may assume $V$ is finite dimensional. Then $\operatorname{Hom}_{k}(V, W)$ has a comodule structure isomorphic to $W \otimes V^{\prime}$. The restriction induces a surjective comodule map

$$
\operatorname{Hom}_{k}(V, W) \rightarrow \operatorname{Hom}_{k}(W, W) \rightarrow 0 \text {. }
$$

Let $\tilde{V}$ be the inverse image of $k I$ which is a subcomodule of $\operatorname{Hom}_{k}(W, W)$. We have a surjective comodule map $\widetilde{V} \rightarrow k \rightarrow 0$ which splits by (2). If $1 \mapsto \psi, k \rightarrow \widetilde{V}$ is a section, $\psi$ is a comodule map $V \rightarrow W$ such that $\psi \mid W=I$.

We show every exact sequence of crossed $B_{q}$-bimodules

$$
0 \rightarrow \tilde{V}_{k, l} \rightarrow V \rightarrow \mathbb{C} \rightarrow 0
$$

splits. The same method applies to $\tilde{V}_{k, l}^{(-)}$, too. It will follow from 7.1 that the largest subcoalgebra $E_{q}$ of the quantum double $\mathscr{E}_{q}=\operatorname{End}_{\mathbb{C}}\left(B_{q}\right)$ is cosemisimple, yielding 5.5 Theorem.

The above exact sequence splits as $B_{q}$-comodules, since $B_{q}$ is cosemisimple. Let $V=\tilde{V}_{k, l} \oplus \mathbb{C} \zeta$ be the decomposition as $B_{q}$-comodules. We have $K \zeta=\zeta, E \zeta=0=F \zeta$ and there are elements $w_{a}, w_{b}, w_{c}, w_{d}$ in $\widetilde{V}_{k, l}$ such that

$$
\zeta a=\zeta+w_{a}, \quad \zeta b=w_{b}, \quad \zeta c=w_{c}, \quad \zeta d=\zeta+w_{d} .
$$

7.2 Lemma. We have

(1) $K w_{a}=w_{a}$, (2) $K w_{b}=q^{-2} w_{b}$, (3) $K w_{c}=q^{2} w_{c}$, (4) $K w_{d}=w_{d}$

(5) $E w_{a}=-q^{2} w_{c}$, (6) $E w_{b}=w_{a}-w_{d}$, (7) $E w_{c}=0$, (8) $E w_{d}=w_{c}$,

(9) $F w_{a}=q w_{b}$, (10) $F w_{b}=0$, (11) $F w_{c}=q^{-1}\left(w_{d}-w_{a}\right)$, (12) $F w_{d}=-q^{-1} w_{b}$,

(13) $w_{b} a=q\left(w_{b}+w_{a} b\right), \quad$ (14) $w_{c} a=q\left(w_{c}+w_{a} c\right)$,

(15) $w_{b} d=q^{-1}\left(w_{b}+w_{d} b\right), \quad$ (16) $w_{c} d=q^{-1}\left(w_{c}+w_{d} c\right)$,

(17) $w_{b} c=w_{c} b$

(18) $w_{a}+w_{d} a=q w_{b} c$, (19) $w_{d}+w_{a} d=q^{-1} w_{b} c$.

Proof. The first 12 relations are consequences of 5.1 applied to $v=\zeta$. The remaining 7 relations follow from the commutation relations of 4.2 .

7.3 Corollary. $w_{b}=0$ if and only if $w_{c}=0$.

Proof. If $w_{b}=0, w_{a}=w_{d}$ by (6), and $w_{c}=-q^{2} w_{c}$ by (5), (8). Hence $w_{c}=0$, since $q$ is real. Similarly, $w_{b}=0$ if $w_{c}=0$.

We will find all non-trivial pairs of elements $w_{b}, w_{c}$ in $\tilde{V}_{k, l}$ satisfying conditions (2), (3), (7), (10), and (17).

The Clebsch-Gordan rule [T2, Proposition 2.4] tells that

$$
\tilde{V}_{k, l} \cong V_{k+l} \oplus V_{k+l-2} \oplus \cdots \oplus V_{|k-l|}
$$

as $B_{q}$-comodules or $U_{q}$-modules. This means the $U_{q}$-module $\tilde{V}_{k, l}$ has a highest 
weight vector of weight 2 (or a lowest weight vector of weight -2 ) if and only if

$$
k \equiv l \bmod 2 \text { and }|k-l| \leqq 2 \leqq k+l .
$$

This condition is satisfied if and only if $(k, l)$ is of the form $(r+1, r+1),(r, r+2)$, or $(r+2, r)$ for some $r \geqq 0$. In any case, we have

$$
r=\frac{k+l}{2}-1
$$

We have a highest weight vector $\sum_{i=0}^{r} x_{i} v_{r-i, l-i}$ of weight 2 (respectively a lowest weight vector $\sum_{i=0}^{r} y_{i} v_{k-i, r-i}$ of weight -2 ), where

$$
\begin{aligned}
x_{i}= & \left(-q^{l}\right)\left(-q^{l-2}\right) \cdots\left(-q^{l-2(i-1)}\right) \\
& \cdot[r][r-1] \cdots[r-(i-1)][k-r+1][k-r+2] \cdots[k-r+i], \\
y_{i}= & \left(-q^{k}\right)\left(-q^{k-2}\right) \cdots\left(-q^{k-2(i-1)}\right) \\
& \cdot[r][r-1] \cdots[r-(i-1)][l-r+1][l-r+2] \cdots[l-r+i] .
\end{aligned}
$$

Let

$$
w_{c}=\lambda \sum_{i=0}^{r} x_{i} v_{r-i, l-i}, \quad w_{b}=\mu \sum_{i=0}^{r} y_{i} v_{k-i, r-i} .
$$

We have by (6.4),

$$
\begin{aligned}
& w_{c} b=-q \lambda\left(q-q^{-1}\right) \sum x_{i}[i+1][l-i] v_{r-i, l-i-1}, \\
& w_{b} c=\mu\left(q-q^{-1}\right) \sum y_{i}[i+1][k-i] v_{k-i-1, r-i} .
\end{aligned}
$$

Case $(k, l)=(r+1, r+1)$. Condition $w_{b} c=w_{c} b$ implies $-q \lambda x_{i}=\mu y_{i}, 0 \leqq i \leqq r$. Since $x_{i}=y_{i}$ in this case, this is equivalent to $\mu=-q \lambda$.

Case $(k, l)=(r, r+2)$ or $(r+2, r)$. In this case, one of $w_{b} c$ and $w_{c} b$ has length $r$, and the other $r+1$. Hence the condition $w_{b} c=w_{c} b$ will imply both are zero. If $r>0$, it follows that $w_{b}=w_{c}=0$. If $r=0$, we have

$$
\begin{array}{lll}
\lambda=0 & \text { if } & k<l, \\
\mu=0 & \text { if } & l<k .
\end{array}
$$

Summarizing the above, we get the following.

7.4 Proposition. The following list gives all non-trivial pairs of elements $w_{b}, w_{c}$ in $\tilde{V}_{k, l}$ satisfying conditions (2), (3), (7), (10), and (17) of 7.2 .

(a) $k=l=r+1$ with $r \geqq 0$ and

$$
w_{b}=-q \lambda \sum_{i=0}^{r} z_{i} v_{r+1-i, r-i}, \quad w_{c}=\lambda \sum_{i=0}^{r} z_{i} v_{r-i, r+1-i}
$$

where

$$
z_{i}=\left(-q^{r+1}\right)\left(-q^{r-1}\right) \cdots\left(-q^{r+1-2(i-1)}\right)[r][r-1] \cdots[r-(i-1)][1][2] \cdots[i+1]
$$


(b) $k=0, l=2, w_{b}=\lambda v_{0,0}, w_{c}=0$,

(c) $k=2, l=0, w_{b}=0, w_{c}=\lambda v_{0,0}$,

where $\lambda \in \mathbb{C}$.

To show that the extension $V=\tilde{V}_{k, l} \oplus \mathbb{C} \zeta$ is trivial, first we reduce to the case $w_{b}=w_{c}=0$. By 7.3 and 7.4, it is enough to consider the case (a) of 7.4. In this case, let

$$
\omega=\sum_{i=0}^{r+1} s_{i} v_{r+1-i, r+1-i}
$$

where

$$
s_{i}=\left(-q^{r+1}\right)\left(-q^{r-1}\right) \cdots\left(-q^{r+1-2(i-1)}\right)[r+1][r] \cdots[r+1-(i-1)][1][2] \cdots[i] .
$$

Then $K \omega=\omega, E \omega=0=F \omega$. Since we have

$$
z_{i}=[r+1]^{-1} s_{i}[r+1-i][i+1]
$$

it follows from (6.4) that

$$
\begin{aligned}
& \omega b=-q\left(1-q^{-2}\right)[r+1] \sum_{i=0}^{r} z_{i} v_{r+1-i, r-i}, \\
& \omega c=\left(1-q^{-2}\right)[r+1] \sum_{i=0}^{r} z_{i} v_{r-i, r+1-i} .
\end{aligned}
$$

Hence, if we use

$$
\zeta^{\prime}=\zeta-\lambda\left(1-q^{-2}\right)^{-1}[r+1]^{-1} \omega
$$

instead of $\zeta$, then we have $\zeta^{\prime} b=0=\zeta^{\prime} c$.

We may assume $w_{b}=w_{c}=0$. We have $w_{a}=w_{d}$ by (6) or (11) of 7.2. Call this $w$. Then $K w=w$ by (1), $E w=F w=0$ by (5), (9), $w b=w c=0$ by (13), (14), and $w a=w d=-w$ by (18), (19). (We refer to conditions of 7.2). If we put

$$
\tilde{\zeta}=\zeta+\frac{1}{2} w
$$

it follows that we have $V=\tilde{V}_{k, l} \oplus \mathbb{C} \tilde{\zeta}$, where $\mathbb{C} \tilde{\zeta}$ is a subbimodule isomorphic to the trivial bimodule $\mathbb{C}$. This shows that every extension of $\mathbb{C}$ by $\widetilde{V}_{k, l}$ is trivial. The same argument applies to $\widetilde{V}_{k, l}^{(-)}$, too. This will finish the proof of 5.5 Theorem.

\section{Appendix. The Braiding Structure}

In this appendix, we discuss the braiding structure on $A_{q}$. Mostly, we work over a general field $k$.

The category of (right) crossed bimodules for a bialgebra $H$ is pre-braided [Y, Theorem 5.2]. This means if $X, Y$ are crossed bimodules, then the map

$$
\mathrm{s}_{X, Y}: X \otimes Y \rightarrow Y \otimes X, x \otimes y \mapsto \sum y_{(0)} \otimes x y_{(1)}
$$

is a bimodule map satisfying the coherence condition

$$
\begin{aligned}
& \mathrm{s}_{X \otimes Y, Z}=\left(\mathrm{s}_{X, Y} \otimes I_{Y}\right)\left(I_{X} \otimes \mathrm{s}_{Y, Z}\right), \\
& \mathrm{s}_{X, Y} \otimes Z \\
& =\left(I_{Y} \otimes \mathrm{s}_{X, Z}\right)\left(\mathrm{s}_{X, Y} \otimes I_{Z}\right) .
\end{aligned}
$$


The map $\mathrm{s}_{X, Y}$ is an isomorphism for all crossed bimodules $X, Y$ if $H$ has a twisted antipode, i.e., if $H^{\text {op }}$ or $H^{\text {cop }}$ is a Hopf algebra [Y, Theorem 7.2]. Then the category of crossed bimodules is a braided category. This is the case if $H$ has a bijective antipode.

On the other hand, the concept of a braided bialgebra was introduced by LarsonTowber [LT] and independently by Hayashi [H]. Let $A$ be a bialgebra. By a braiding on $A$, we mean a unit in the algebra $(A \otimes A)^{\prime}$ which we identify with a bilinear map

$$
\langle,\rangle: A \otimes A \rightarrow k
$$

satisfying the following conditions:

$$
\begin{aligned}
\sum\left\langle x_{(1)}, y_{(1)}\right\rangle x_{(2)} y_{(2)} & =\sum\left\langle x_{(2)}, y_{(2)}\right\rangle y_{(1)} x_{(1)}, \\
\langle x y, z\rangle & =\sum\left\langle x, z_{(1)}\right\rangle\left\langle y, z_{(2)}\right\rangle, \\
\langle x, y z\rangle & =\sum\left\langle x_{(2)}, y\right\rangle\left\langle x_{(1)}, z\right\rangle, \\
\langle x, 1\rangle & =\langle 1, x\rangle=\varepsilon(x)
\end{aligned}
$$

for all $x, y, z$ in $A$.

If $A$ is a braided bialgebra, then $\mathrm{Com}^{-A}$, the category of (right) $A$-comodules, is braided. If $V, W$ are $A$-comodules, then the braiding is given by

$$
\mathrm{s}_{V, W}: V \otimes W \rightarrow W \otimes V, v \otimes w \mapsto \sum\left\langle v_{(1)}, w_{(1)}\right\rangle w_{(0)} \otimes v_{(0)} .
$$

Conversely, if the category $\mathrm{Com}^{-A}$ is braided, there is a unique braiding on $A$ such that the braiding of $\mathrm{Com}^{-A}$ is given by (8.3) [LT, Proposition 2.13]. In other words, there is a $1-1$ correspondence between braidings on $A$ and braidings on $\mathrm{Com}^{-\boldsymbol{A}}$.

Let $H$ be a Hopf algebra with bijective antipode, and let $A$ be a subbialgebra of $\mathscr{E}=\operatorname{End}_{k}(H)$, the quantum double. The bialgebra $A$ has the following braiding

$$
\langle x, y\rangle=\left\langle\pi_{2}(x), \pi_{1}(y)\right\rangle, \quad x, y \in A,
$$

where we use the topological Hopf algebra maps of (2.5) and the canonical pairing between $H^{\prime}$ and $H$. Note that $\mathrm{Com}^{-A}$ is identified with a sub-monodial category of the category of crossed $H$-bimodules.

8.5 Proposition. The braiding on $\mathrm{Com}^{-A}$ given by (8.4) and (8.3) coincides with the one induced from the braiding structure of the category of crossed H-bimodules.

Proof. Let $V, W$ be $A$-comodules. Note that

(respectively

$$
v \mapsto \sum v_{(0)} \otimes \pi_{2}\left(v_{(1)}\right), \quad v \in V,
$$

$$
\left.w \mapsto \sum w_{(0)} \otimes \pi_{1}\left(w_{(1)}\right), w \in W\right)
$$

gives the $H$-module structure on $V$ (respectively the $H$-comodule structure on $W$ ). Hence we have

$$
\sum\left\langle\pi_{2}\left(v_{(1)}\right), \pi_{1}\left(w_{(1)}\right)\right\rangle w_{(0)} \otimes v_{(0)}=\sum w_{(0)} \otimes v \pi_{1}\left(w_{(1)}\right),
$$

$v \in V, w \in W$. This means the braiding $\mathrm{s}_{V, W}(8.3)$ coincides with the braiding $\mathrm{s}_{X, Y}$ (8.1) if $X, Y$ denote the crossed bimodules identified with $V, W$. 
We are in this situation if we take $H=B_{q}$ and $A=A_{q}$. In this case, the braiding (8.4) is defined by using the Hopf algebra maps

$$
\pi_{1}: A_{q} \rightarrow B_{q}, \quad \pi_{2}: A_{q} \rightarrow C_{q}
$$

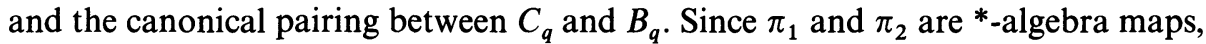
too, it follows from the definition of $*$ on $B_{q}^{\prime}$ (above 4.1 ) that we have

$$
\left\langle x^{*}, y\right\rangle=\overline{\left\langle x, S(y)^{*}\right\rangle}, \quad x, y \in A_{q} .
$$

Since $\langle x, y\rangle=\langle S(x), S(y)\rangle$ [LT, Proposition 2.9] and $S(y)^{*}=S^{-1}\left(y^{*}\right)$, it follows that

$$
\left\langle x^{*}, y^{*}\right\rangle=\overline{\langle S(x), y\rangle}=\overline{\left\langle x, S^{-1}(y)\right\rangle}, \quad x, y \in A_{q} .
$$

In a word, $A_{q}$ is a braided ${ }^{*}$-Hopf algebra.

Acknowledgements. I am grateful to T. Kotera, R. Blattner, S. Montgomery, and P. Podles. Kotera first called my attention to the work [PW]. Blattner and Montgomery invited me to give a lecture on this subject. At that time, the conjecture was unsolved. Podles visited Tsukuba in February 1991 and had useful discussions with me. 5.4 Theorem was obtained before his arrival, and 5.5 Theorem soon after he left.

\section{References}

[APW] Anderson, H., Polo, P., Wen, K.: Representations of quantum algebras, preprint

[D] Drinfeld, V.: Quantum groups. Proc. of ICM-86. Berkeley, CA, USA, pp. 793-820

[H] Hayashi, T.: Quantum groups and quantum determinants, preprint

[LT] Larson, R., Towber, J.: Two dual classes of bialgebras related to the concepts of "quantum group" and "quantum Lie algebra," preprint

[MMNNU] Masuda, T., Mimachi, K., Nakagami, Y., Noumi, M., Ueno, K.: Representations of quantum groups and a $q$-analogue of orthogonal polynomials. C.R. Acad. Sci. Paris, t.307, Series I, 559-564 (1988)

[PW] Podleś, P., Woronowicz, S.: Quantum deformation of Lorentz group. Commun. Math. Phys. 130, 381-431 (1990)

[S] Sweedler, M.: Hopf algebras. New York: W.A. Benjamin, 1969

[T1] Takeuchi, M.: Topological coalgebras. J. Algebra 97, 505-539 (1985)

[T2] Takeuchi, M.: Hopf algebra techniques applied to the quantum group $\mathrm{U}_{q}(\mathfrak{s I}(2))$ Contemp. Math. (to appear)

[W] Woronowicz, S.: Twisted SU(2) group. Publ. RIMS Kyoto University 23, 117-181 (1987)

[Y] Yetter, D.: Quantum groups and representations of monoidal categories. Math. Proc. Camb. Phil. Soc. 108, 261-290 (1990)

Communicated by A. Connes 